\title{
Severe maternal morbidity and maternal near miss in the extremes of reproductive age: results from a national cross- sectional multicenter study
}

\author{
Fernando César Oliveira Jr $^{1,2^{\wedge}}$, Fernanda Garanhani Surita ${ }^{1 *}$, João Luiz Pinto e Silva ${ }^{1}$, José Guilherme Cecatti ${ }^{1}$, \\ Mary Angela Parpinelli', Samira M Haddad ${ }^{1}$, Maria Laura Costa ${ }^{1}$, Rodolfo Carvalho Pacagnella ${ }^{1}$, \\ Maria Helena Sousa ${ }^{3}$, and João Paulo Souza ${ }^{3}$ for The Brazilian Network for Surveillance of Severe Maternal Morbidity \\ Study Group
}

\begin{abstract}
Background: The aim of this study was to assess severe maternal morbidity (SMM) and near miss (NM) cases among adolescent girls and women over 35 years of age in the Brazilian Network for Surveillance of Severe Maternal Morbidity, using a set of standard criteria, compared to pregnant women aged 20 to 34 years.

Methods: A cross-sectional multicenter study conducted in 27 referral obstetric units in Brazil. All pregnant women admitted to these centers during a one-year period of prospective surveillance were screened to identify cases of maternal death (MD), NM and other SMM. Indicators of maternal morbidity and mortality were evaluated for the three age groups. Sociodemographic, clinical and obstetric characteristics, gestational and perinatal outcomes, main causes of morbidity and delays in care were also compared. Two multiple analysis models were performed, to estimate the adjusted prevalence ratio for identified factors that were independently associated with the occurrence of severe maternal outcome $(S M O=M N M+M D)$.
\end{abstract}

Results: Among SMM and MD cases identified, the proportion of adolescent girls and older women were $17 \%$ each. The risk of MNM or death was 25\% higher among older women. Maternal near miss ratio and maternal mortality ratios increased with age, but these ratios were also higher among adolescents aged 10 to 14, although the absolute numbers were low. On multivariate analysis, younger age was not identified as an independent risk factor for SMO, while this was true for older age (PR 1.25; 1.07-1.45).

Conclusions: SMO was high among women below 14 years of age and increased with age in Brazilian pregnant women.

Keywords: Maternal near-miss, Obstetric complication, Maternal death, Maternal age, Extremes of reproductive age, Maternal morbidity

\section{Background}

Maternal mortality is a key heath care indicator related to a country's level of development. Adolescent girls are most vulnerable to social maladjustment. This may be the cause or result of unexpected pregnancies, which increase the occurrence of unsafe abortions, the discontinuation of

\footnotetext{
* Correspondence: surita@unicamp.br

Deceased

'Department of Obstetrics and Gynecology, University of Campinas, Rua Alexander Fleming, 101, 13083-881 Campinas, SP, Brazil

Full list of author information is available at the end of the article
}

formal education and are associated with lower adherence to prenatal care [1]. In a similar fashion, although for distinct reasons, older pregnant women are also considered at high risk of obstetric complication due to a higher prevalence of associated morbid conditions and/or multiparity $[2,3]$.

The study of maternal death (MD) is fraught with difficulties and therefore, in the past years, women who survive severe conditions of pregnancy or near-miss (NM) complications have attracted great interest as a source of information on processes that can lead to death. The 
investigation of these cases can be used as a complementary method to audits and inquiries into $\mathrm{MD}[4,5]$. The investigation of NM events provides important details on factors that may contribute to both MD and NM. Knowledge on the prevalence and causes of NM may indeed constitute a new form of evaluating obstetric care [6,7].

In 2009, the World Health Organization (WHO) defined Maternal Near-Miss as a woman who almost died but survived a complication that occurred during pregnancy, childbirth or within the 42 days following pregnancy termination. Standardized criteria for NM identification have been defined, according to organ dysfunction and/ or failure- and the WHO recommends that this approach should be used to assess quality of obstetric care $[6,8,9]$. Clinical signs, laboratory tests and management interventions were used, aimed at diagnosing organ dysfunction or failure. These criteria were previously validated in a Brazilian obstetric population [10]. However, in the published literature a wide variety of criteria used to identify cases of maternal NM still prevail [11].

Although pregnancy during adolescence is a constant public health issue, due to the recognized higher risk for the mother and infant, in addition to the strong biological, psychological and social impact, maternal death associated with adolescence is more well-known than the corresponding morbidity. The risk of maternal death for women aged 15 to 19 years is twice the risk for women aged 20 to 24 years. For those aged 10 to 15 years, the risk of death may be even five-fold higher, compared to women aged 20 to 24 years [12]. Half of all adolescent births in the world occur in only seven countries: Bangladesh, Brazil, Democratic Republic of Congo, Ethiopia, India, Nigeria and the United States [13]. Apart from the United States, the remaining six countries still have a high maternal mortality ratio. Furthermore, pregnancies among women aged over 35 years are increasing worldwide. In Finland, the rate of old age pregnancy increased from a total of $16.7 \%$ in 1997 to $19.2 \%$ in 2007 [14]. Data from the WHO for developing countries around the world reveal a prevalence of $10.6 \%$ of pregnancies in women aged over 35 years between 2004 and 2008 [15]. Pregnancies in older women are also associated with a higher maternal and perinatal morbidity and mortality, with a greater risk of various morbid conditions, such as hypertension and diabetes, anomalous presentation, intrapartum fetal distress, cesarean delivery and postpartum hemorrhage. In older pregnant women following delivery, Apgar scores were lower and there was a higher incidence of low birth weight and preterm when compared to younger women $[2,3,16,17]$.

However, information on the occurrence of near-miss and severe maternal morbidity in both extremes of reproductive age is scarce. The lack of knowledge considering the impact of the problem and the existence of any associated factors liable to intervention makes it difficult to improve healthcare and prevent complications in these age groups. Thus, the aim of the current study is to explore the characteristics of severe maternal morbidity, with special focus on the extremes of reproductive age, among women identified in the Brazilian Network for Surveillance of Severe Maternal Morbidity. Knowledge of these features may lead to improvement in public policies and result in better care of women developing severe maternal morbidity in the extremes of reproductive ages.

\section{Methods}

Detailed information on the study's method and procedures were previously published $[5,18]$. Briefly, this was a multicenter cross-sectional study, implemented in 27 referral obstetric units in all geographical regions in Brazil. From July 2009 to June 2010, a prospective surveillance was carried out and data was collected to identify cases of potentially life-threatening maternal conditions (PLTC), maternal near-miss and maternal death, using the new WHO's concept and criteria [8]. The present article refers to an analysis focused on the occurrence of severe maternal morbidity $(\mathrm{SMM}=\mathrm{PLTC}+\mathrm{MNM}+\mathrm{MD})$ and severe maternal outcome $(\mathrm{SMO}=\mathrm{MNM}+\mathrm{MD})$ related to the extremes of maternal age.

Sample size calculation determined that about 75,000 deliveries should be surveyed to identify around 750 near-miss cases, using an approximate theoretical incidence of 10 near miss cases per 1000 deliveries as basis for calculation [6].

Maternal age was considered an independent variable that was categorized into three groups: adolescence (between 10 and 19 years, with a subgroup aged 10-14 years and another aged 15-19 years), pregnant women over age 35 (with a subgroup aged 35-39 years and another aged 40-49 years), and a reference group, aged between 20 and 34 years. Data concerning sociodemographic characteristics, obstetric history, previous morbid conditions, prenatal care and obstetric complications were gathered. The occurrence of potentially life-threatening conditions (PLTC-hemorrhagic, hypertensive, infectious and other complications), Maternal Near-Miss (MNMclinical/laboratory criteria and management of severity) and Maternal Death (MD) was assessed, according to the WHO definition [8], in addition to data on pregnancy termination and perinatal results. The occurrence of delays in obstetric care was also assessed. For this purpose, specific questions in the questionnaire addressed delays for women seeking care, for reaching the health facility and also for obtaining the adequate and timely treatment for their complications. In addition specific rules for checking the occurrence of these delays were developed and systematically used for all cases. 
Data was initially collected in a form following a oneyear prospective surveillance by a research coordinator in each center. Subsequently, after the data was checked by the local investigator it was entered and stored in the platform OpenClinica version 2.5.5 (Akaza Research, Waltham, MA, USA). Digital data was stored in a protected database from a computer accessible from the institutional website of the coordinating center of the study. Control of data quality was performed for every case in each participating center by using local rules and also in the coordinating center using general rules of completeness and consistency. Trained personnel from the coordinating center performed site monitoring visits for randomly selected cases (around 5\% of those identified), cross-checking local data with the information already stored in the system. A manual of operation was developed and used by all of the centers involved in the study, in order to standardize concepts and data collection [18].

Sampling design corresponded to a single-stage cluster sampling, with 27 Primary Sampling Units (PSU), relative to the 27 centers (hospitals). The sampling design did not involve PSU stratification or data weighting because all centers were secondary or tertiary units with relatively similar teaching hospitals caring for at least 1000 deliveries per year. The unit of analysis was the medical record of each hospitalized woman with a PLTC (potentially life-threatening condition), MNM (maternal near-miss) or MD (maternal death). All variables studied had relatively low ICC values (intraclass correlation coefficients), demonstrating the necessary heterogeneity among study clusters. Specifically for the age variable, the ICC was 0.013 (95\% CI 0.002-0.024) [19].

\section{Data analysis}

Data analysis initially consisted of the distribution of women identified as PLTC, MNM and MD by age groups, obtaining prevalence ratios (PR) and respective confidence intervals ( $95 \% \mathrm{CI}$ ) adjusted by cluster effect of the study. Age groups were tested as a risk factor for the occurrence of MNM or MD. Information was not collected for women without maternal complications and therefore the distribution of live births for the study period according to maternal age categories was not possible. As a proxy, the number of live births (LB) was estimated according to the number of LB by maternal age group informed for the country in 2009 [20] so that health indicators related to maternal morbidity and mortality could be estimated: MNMR (maternal near-miss ratio per 100,000 LB), SMOR (severe maternal outcome ratio per 1,000 LB), MMR (maternal mortality ratio per 1,000 LB) and MNM:MD (the ratio between MNM and MD) [8]. Subsequently, the distributions of variables relative to socio demographic and clinical characteristics, obstetric history, habits, time and mode of pregnancy termination, neonatal results, and main causes of severe maternal morbidity by the three age groups currently analyzed. The differences between groups were evaluated by the $\chi^{2}$ test.

Finally, Poisson multiple regression analysis was used to identify factors that were independently and significantly associated with the worst outcomes (MD or MNM), compared to PLTC, for two models. The first model included only females aged 10 to 34 years (10-19 vs 20-34) for evaluating adolescent pregnant women, and the second model only women aged 20 to 49 years (20-34 vs 35-49) for the evaluation of older women. Women aged 20 to 34 years served as the reference group category in both models. The estimated coefficient, standard error (SE) of the coefficient, descriptive level (p) and prevalence ratio adjusted to cluster effect and remaining model variables, with their respective confidence interval ( $\left.\mathrm{PR}_{\mathrm{adj}}[95 \% \mathrm{CI}]\right)$ were obtained. For building the models for multiple analyses, all the predictive variables initially entered and then they were manually selected through the backward criteria in the Stata software v.7. Statistical analysis was carried out with software SPSS $^{\oplus}$ version 17.0 (SPSS, Chicago, IL, USA) and Stata version 7.0 (StataCorp, College Station, Tx, USA). The descriptive level $(\alpha)$ was preset at 5\% (95\% confidence level) and a single-stage conglomerate sampling design was considered.

The study was previously approved by the Institutional Review Board (IRB of FCM/UNICAMP) of each center and by the National Research Committee prior to study initiation on May 5th 2009 (CEP 027/2009). This study did not require informed consent because data were collected exclusively from medical records immediately after patient discharge.

\section{Results}

During the 12 months of the study, 9555 PLTC, MNM or MD cases were identified among 82,144 live births occurring in these hospitals during this period. Adolescents were responsible for 1713 (17.9\%) of these cases, while older women aged 35 to 49 years accounted for a similar number of cases (1622-17\%).

Table 1 shows a 1.25-fold higher risk of maternal near miss and maternal death for the 35-49 year age group (PR 1.25, 95\% CI: 1.07-1.45), which was even higher in the 40-49 year age group (PR 1.52, 95\% CI: 1.19-1.93). The prevalence ratio for near miss and maternal death was not higher for the total number of adolescents, although when ages 10 to 14 years were considered, the occurrence of 1 maternal death (MD) for every 2.3 MNN cases attracted attention. In the reference group, 1 death occurred (MD) for every 5.1 MNM cases. Table 1 also shows the MNMR and MMR values that increase with increasing age starting at the 15-19 year group, but 
Table 1 Distribution of women by age group and PLTC, MNM and MD

\begin{tabular}{cccccccccccc}
\hline Age (years) & $\%$ & PLTC & MNM & MD & $\begin{array}{c}\text { PR (SMO: } \\
\text { MNM + MD) }\end{array}$ & 95\% Cl & ELB & MNMR/1000LB & SMOR/1000LB & MMR/100000 LB & MNM:MD \\
\hline $10-19$ & 17.9 & 18.2 & 15.2 & 15.72 & 0.89 & $0.77-1.04$ & 16,388 & 7.14 & 8.48 & 134.2 & $5.3: 1$ \\
$10-14$ & 1.2 & 1.2 & 0.9 & 2.13 & 0.97 & $0.57-1.65$ & 797 & 8.78 & 12.55 & 376.4 \\
$15-19$ & 16.8 & 17.0 & 14.3 & 13.19 & 0.88 & $0.76-1.03$ & 15,591 & 7.06 & 8.27 & 121.9 & 5.3 .1 \\
$20-34$ & 65.1 & 65.3 & 62.6 & 67.95 & 1.00 & Ref & 57,435 & 8.39 & 10.05 & 165.4 & $5.1: 1$ \\
$35-49$ & 17.0 & 16.5 & 22.2 & 16.23 & 1.25 & $1.07-1.45$ & 8,321 & 20.55 & 23.31 & 276.4 & $7.4: 1$ \\
$35-39$ & 12.1 & 11.8 & 14.8 & 10.7 & 1.19 & $1.00-1.41$ & 6,506 & 17.52 & 19.83 & 230.6 & $7.6: 1$ \\
$40-49$ & 4.9 & 4.7 & 7.4 & 5.7 & 1.52 & $1.19-1.93$ & 1,815 & 31.40 & 35.81 & 440.8 & $7.1: 1$ \\
Total & 9,555 & 8,645 & 770 & 140 & & & 82,144 & 9.37 & 11.08 & 170.4 & $5.5: 1$
\end{tabular}

ELB: estimated live births; LB: number of live births; MD: Maternal Death; MMR: maternal mortality ratio; MNM: Maternal Near Miss; MNM:MD: maternal near miss to mortality ratio; MNMR: maternal near miss ratio; PLTC: Potentially Life Threatening Condition; SMOR: severe maternal outcome ratio; PR: prevalence ratio. The number of ELB in each age group was obtained with the number of total live births in the period by age group distribution, according to data from SINASC (Brazil, 2009).

Maternal near miss ratio (MNMR), severe maternal outcome ratio (SMOR), maternal mortality ratio (MMR) and maternal near miss to mortality ratio (MNM:MD). Brazil, 2009-2010

with values also higher for the extreme adolescent group (girls aged 10-14 years).

Table 2 shows the difference between age groups regarding sociodemographic characteristics and clinical/ obstetric history. Adolescents had a significantly higher proportion of non-whites, low school education, lack of a steady partner, low birthweight and nulliparity (only $12 \%$ of these reported having a previous pregnancy). In contrast, women over 35 years of age, compared to the reference group, had a higher proportion of low schooling, steady relationship, obesity, history of morbid clinical conditions (hypertension, diabetes, heart and thyroid disease), multiparity, history of previous abortions/cesarean sections and a lower number of prenatal visits.

In general, adolescents had a better perinatal result (Table 3). These women had a significantly lower number of preterm deliveries, cesarean sections and low birth weight infants, and a higher number of vaginal deliveries, live births that were discharged in good conditions compared to the control group. In contrast, women over 35 years had a significantly higher number of cesarean sections and stillbirths than controls.

Inregards to the conditions identified as main causes of severe morbidity, the only differences found among the age groups were a lower proportion of hemorrhagic causes and a higher proportion of hypertensive causes among adolescent women. Furthermore, adolescents also had a higher proportion of situations identified as delays in obtaining adequate care for the complication (Table 4).

The multivariate analyses model, including only adolescents and the reference group, showed that women with infectious, clinical/surgical and bleeding conditions (between three and five-fold) and who suffered any delay in care (two-fold) exhibited a higher risk of having SMO (MNM and MD). The risk was also higher when there were preexisting clinical conditions such as diabetes, neoplasm and others, in non-white skin color (between 1.5 and 2-fold). In contrast, obesity decreased by almost $50 \%$ the risk of MNM and MD. In this analysis, however, age was not identified as a factor associated with a worse case outcome (Table 5).

The same analysis including only women aged 35 or older and the control group also showed a higher risk of having SMO (MNM or MD) due to infectious, clinical/ surgical and bleeding conditions, and whether there was any delay in care (two to five-fold). Among the preexisting clinical conditions, kidney disease, diabetes, thalassemia/sickle cell anemia and others, in addition to drug addiction and having received prenatal care in another health service, were also greater risk factors for a worse outcome. It is also observed that previous obesity and lack of a steady partner were protective factors. Only in this analysis increased maternal age was shown to significantly increase by $25 \%$ the risk of a worse outcome for MNM or MD (Table 6).

\section{Discussion and conclusions}

It is well-known that maternal mortality risk is higher in two periods of age. The first is at the beginning of reproductive life or adolescence, going through a decrease and stabilization, and increasing again at the end of reproductive life [21]. The current analysis was carried out to assess factors that may be associated with a worse outcome of maternal complications and to evaluate whether the occurrence of NM also follows a pattern similar to that of maternal death regarding female age, in the context of a national surveillance study of severe maternal morbidity in Brazil.

The present study showed virtually the same proportion of cases with severe complications in both extremes of reproductive life (17\%), in addition to almost $12 \%$ of repeat pregnancies in adolescence among those with 
Table 2 Distribution of women with any severe maternal morbidity by age group according to some characteristics, clinical and obstetric history

\begin{tabular}{|c|c|c|c|c|c|}
\hline Characteristics & $10-19$ years & $\mathrm{p}^{*}$ & $20-34$ years & $p^{* *}$ & $35-49$ years \\
\hline Ethnicity (non-white) (n) & $60.8(1,259)$ & 0.022 & $56.9(4,675)$ & 0.912 & $56.7(1,205)$ \\
\hline Schooling (up to primary school) (n) & $58.0(1,275)$ & $<0.001$ & $41.6(4,514)$ & $<0.001$ & $52.9(1,134)$ \\
\hline Marital status (No steady partner) (n) & $62.3(1,455)$ & $<0.001$ & $45.2(5,255)$ & $<0.001$ & $36.6(1,329)$ \\
\hline $\mathrm{BMI}(\mathrm{n})$ & $(630)$ & $<0.001$ & $(2,600)$ & 0.014 & $(704)$ \\
\hline Low weight & 30.0 & & 13.4 & & 9.8 \\
\hline Adequate & 33.8 & & 27.1 & & 24.9 \\
\hline Overweight & 19.5 & & 27.7 & & 28.4 \\
\hline Obesity & 16.7 & & 31.8 & & 36.9 \\
\hline Previous condition ${ }^{\&}(\mathrm{n})$ & $29.3(1,446)$ & $<0.001$ & $49.6(5,363)$ & $<0.001$ & $66.1(1,432)$ \\
\hline Hypertension & 3.5 & $<0.001$ & 16.7 & $<0.001$ & 35.4 \\
\hline Diabetes mellitus & 0.6 & $<0.001$ & 2.2 & $<0.001$ & 5.7 \\
\hline Cardiac & 2.1 & 0.287 & 2.7 & $<0.001$ & 4.7 \\
\hline Thyroid disease & 0.6 & 0.028 & 1.3 & 0.010 & 2.8 \\
\hline Respiratory & 3.1 & 0.458 & 2.8 & 0.776 & 2.7 \\
\hline Nephropathy & 0.6 & 0.061 & 1.4 & 0.906 & 1.4 \\
\hline Thalassemia/sickle cell anemia & 0.6 & 0.148 & 1.0 & 0.012 & 0.3 \\
\hline Collagenosis & 0.4 & 0.539 & 0.6 & 0.508 & 0.5 \\
\hline Neoplasms & 0.1 & 0.119 & 0.3 & 0.641 & 0.4 \\
\hline HIV/AIDS & 0.3 & $<0.001$ & 1.3 & 0.973 & 1.3 \\
\hline Low weight & 1.0 & $<0.001$ & 0.2 & 0.794 & 0.1 \\
\hline Obesity & 12.9 & $<0.001$ & 25.8 & 0.033 & 29.2 \\
\hline Smoking & 3.9 & 0.013 & 5.9 & 0.599 & 6.4 \\
\hline Drug addiction & 0.9 & 0.153 & 1.3 & 0.934 & 1.3 \\
\hline Other & 3.4 & 0.100 & 4.9 & 0.003 & 8.3 \\
\hline Parity (n) & $(1,707)$ & $<0.001$ & $(6,171)$ & $<0.001$ & $(1,615)$ \\
\hline 0 & 88.2 & & 44.8 & & 18.8 \\
\hline $1-2$ & 11.4 & & 43.4 & & 47.8 \\
\hline$\geq 3$ & 0.4 & & 11.7 & & 33.4 \\
\hline Previous abortion & $7.3(1,707)$ & $<0.001$ & $22.5(6,169)$ & $<0.001$ & $37.6(1,615)$ \\
\hline Previous Cesarean section & $5.6(1,703)$ & $<0.001$ & $25.9(6,084)$ & $<0.001$ & $36.8(1,572)$ \\
\hline Number of prenatal visits & $(1,334)$ & $<0.001$ & $(4,863)$ & 0.086 & $(1,260)$ \\
\hline 0 & 5.4 & & 8.6 & & 10.2 \\
\hline $1-5$ & 41.9 & & 37.0 & & 38.5 \\
\hline$\geq 6$ & 52.7 & & 54.4 & & 51.3 \\
\hline
\end{tabular}

*p-values for proportions between adolescent and control groups.

**p-values for proportions between older age and control groups.

\&previous conditions are not mutually exclusive.

Brazil, 2009-2010.

SMM. As expected, the study also showed very specific differences between both extremes of age.

Among the total number of adolescents, the risk of SMO (NM or MD) was not significantly higher. However, in adolescents up to age 14, the extremely low case/fatality ratio captures attention. If adolescent pregnancy is already considered a problem [1], the problem is compounded especially in the 10-14 year age group [12]. All unfavorable conditions are exacerbated: physical, social, economic or violent incidents. Although the ratio of cases identified in this specific age group was small $(1.2 \%$ of the total number of cases studied), not allowing a deeper analysis, a high risk was observed, reinforcing the need to expend sustained efforts to avoid pregnancy in this age period [12]. 
Table 3 Distribution of women with any severe maternal morbidity by age group according to time and mode of pregnancy termination and neonatal outcomes

\begin{tabular}{|c|c|c|c|c|c|}
\hline Outcomes & $10-19$ years & p-value* & $20-34$ years & p-value** & $35-49$ years \\
\hline Preterm birth <37 weeks (a) & 36.5 & $<0.001$ & 47.3 & 0.116 & 49.7 \\
\hline (n) & $(1,510)$ & & $(5,444)$ & & $(1,381)$ \\
\hline
\end{tabular}

Mode or pregnancy termination (b)

$<0.001$

0.037

\section{Vaginal birth \\ Cesarean section \\ Abortion/ectopic \\ Still pregnant}

(n)

Apgar 5 th $\min <7$ (c)

(n)

Birth weight $<2.500 \mathrm{~g}(\mathrm{~d})$

(n)

Neonatal condition at birth (e)

$$
\begin{aligned}
& \text { Live birth } \\
& \text { Stillbirth }
\end{aligned}
$$

(n)

Neonatal outcome $(f)$

$$
\begin{aligned}
& \text { Discharge } \\
& \text { Admitted or transferred } \\
& \text { Neonatal death }
\end{aligned}
$$

(n)

(a) 1220 cases excluded (still pregnant, abortion, ectopic pregnancy, or missing GA)

(b) 41 cases excluded (missed information on mode of pregnancy termination).

(c) 1930 cases excluded (still pregnant, abortion, ectopic pregnancy, fetal death, or missing Apgar).

(d) 1632 cases excluded (still pregnant, abortion, ectopic pregnancy, or missing weight).

(e) 1434 cases excluded (still pregnant, abortion, ectopic pregnancy, or missing condition at birth).

\begin{tabular}{|c|c|c|}
\hline 21.7 & & 19.6 \\
\hline 65.1 & & 65.2 \\
\hline 6.1 & & 7.4 \\
\hline 7.0 & & 7.8 \\
\hline$(6,194)$ & & $(1,619)$ \\
\hline 3.9 & 0.863 & 3.8 \\
\hline$(4,954)$ & & $(1,241)$ \\
\hline 41.0 & 0.274 & 42.9 \\
\hline$(5,147)$ & & $(1,308)$ \\
\hline
\end{tabular}

(f) 2109 cases excluded (still pregnant, abortion, ectopic pregnancy, or missing neonatal outcome).

* $p$-values for proportions between adolescent and control groups.

** $p$-values for proportions between older age and control groups.

Brazil, 2009-2010.
0.019

$\begin{array}{cc}95.2 & 93.4 \\ 4.7 & 6.6 \\ (5,274) & (1,348)\end{array}$

0.370

$74.2 \quad 75.2$

$23.0 \quad 22.7$

\begin{tabular}{|c|c|c|c|c|c|}
\hline Main causes of SMM & $10-19$ years & p-value* & $20-34$ years & p-value ${ }^{* *}$ & $35-49$ years \\
\hline Hemorrhage & 19.4 & $<0.002$ & 24.7 & 0.837 & 25.1 \\
\hline Hypertension & 73.0 & 0.009 & 69.3 & 0.555 & 70.6 \\
\hline Infections & 1.3 & 0.177 & 1.0 & 0.464 & 1.1 \\
\hline Clinical surgical conditions & 11.0 & 0.898 & 10.9 & 0.457 & 9.9 \\
\hline (n) & $(1,713)$ & & $(6,220)$ & & $(1,622)$ \\
\hline Any delay (a) & 57.7 & 0,002 & 52.7 & 0.503 & 53.9 \\
\hline (n) & $(1550)$ & & $(5,686)$ & & $(1,480)$ \\
\hline
\end{tabular}

$2.8-2.1$

$(4,839) \quad(1,212)$

Table 4 Distribution of women with any severe maternal morbidity by age group according to main determining causes and delays in appropriate care

(a) 839 cases excluded (missing information on delays).

*p-values for proportions between adolescent and control groups.

**p-values for proportions between older age and control groups.

Brazil, 2009-2010. 
Table 5 Conditions independently associated with higher severity (SMO: MNM + MD) among women aged 10 to 19 compared to aged 20 to 34 years [ $=5,350]$

\begin{tabular}{lcccc}
\hline Variables & Coefficient & SE coeff. & p & PR $_{\text {adj }}[\mathbf{9 5 \%}$ Cl] \\
\hline Infectious conditions & 1.66 & 0.33 & $<0.001$ & $5.23[2.64-10.36]$ \\
Clinical surgical conditions & 1.52 & 0.16 & $<0.001$ & $4.58[3.33-6.31]$ \\
Hemorrhagic conditions & 1.01 & 0.28 & $<0.002$ & $2.75[1.54-4.89]$ \\
Any delay & 0.75 & 0.12 & $<0.001$ & $2.11[1.64-2.72]$ \\
Another previous condition & 0.71 & 0.11 & $<0.001$ & $2.03[1.60-2.57]$ \\
Previous condition: diabetes & 0.63 & 0.20 & 0.003 & $1.88[1.26-2.82]$ \\
Previous condition: neoplasms & 0.53 & 0.22 & 0.024 & $1.71[1.08-2.69]$ \\
Ethnicity (nonwhite) & 0.31 & 0.14 & 0.041 & $1.36[1.01-1.82]$ \\
Previous condition: obesity & -0.65 & 0.17 & $<0.002$ & $0.52[0.37-0.74]$ \\
Constant & -3.62 & 0.21 & $<0.001$ & \\
\hline
\end{tabular}

$\mathrm{PR}_{\mathrm{adj}}$ : prevalence ratio adjusted by cluster effect and also by variables shown to be significant in the final model.

Poisson multiple regression, controlled by: Age 1 (10-19 years: 1/20-34: 0); Ethnicity (Nonwhite: 1/Other: 0); Schooling (up to primary school: 1/Other: 0); Marital status (no steady partner: 1/Other: 0); BMI (Low weight, adequate: 0/overweight, obesity: 1); Prenatal care at the same service (Absent: $1 /$ Present: 0); Previous clinical conditions (Yes: 1/No: 0); Chronic hypertension (Yes: 1/No: 0); Obesity (Yes: 1/No: 0); Low weight (Yes: 1/No: 0); Diabetes mellitus (Yes: 1/No: 0); Smoking (Yes: 1/No: 0); Cardiac diseases (Yes: 1/No: 0); Respiratory diseases (Yes: 1/No: 0); Renal diseases (Yes: 1/No: 0); Thalassemia/sickle cell anemia (Yes: 1/No: 0); HIV/ AIDS (Yes: 1/No: 0); Thyroid diseases (Yes: 1/No: 0); Neurologic diseases/epilepsy (Yes: 1/No: 0); Collagenosis (Yes: 1/No: 0); Neoplasms (Yes: 1/No: 0); Other preexisting condition (Yes: 1/No: 0); Drug addiction (Yes: 1/No: 0); Parity (0/ $\geq 1$ : 1); Previous abortion (Yes: 1/No: 0); Previous Cesarean section (Yes: 1/No: 0); Time since last delivery (years); Number of prenatal visits (up to 5: 0/ $\geq 6$ : 1); hemorrhagic conditions (Yes: 1/No: 0); Hypertensive conditions (Yes: 1/No: 0); Infectious conditions (Yes: 1/No: 0); Clinical surgical conditions (Yes: 1/No: 0); Any delay (Yes: 1/No: 0). Brazil, 2009-2010.

Table 6 Conditions independently associated with higher severity (SMO: MNM + MD) among women aged 35 to 49 compared to women aged 20 to 34 years $[n=5,331]$

\begin{tabular}{lcccc}
\hline Variables & Coefficient & SE coeff. & P & PR $_{\text {adj }}[\mathbf{9 5 \%}$ Cl] \\
\hline Infectious conditions & 1.55 & 0.26 & $<0.001$ & $4.71[2.75-8.07]$ \\
Clinical surgical conditions & 1.37 & 0.19 & $<0.001$ & $3.94[2.68-5.80]$ \\
Hemorrhagic conditions & 1.12 & 0.26 & $<0.001$ & $3.06[1.81-5.18]$ \\
Any delay & 0.71 & 0.09 & $<0.001$ & $2.03[1.70-2.42]$ \\
Another previous condition & 0.80 & 0.12 & $<0.001$ & $2.23[1.75-2.84]$ \\
Previous condition: renal diseases & 0.78 & 0.22 & $<.002$ & $2.17[1.39-3.40]$ \\
Previous condition: diabetes & 0.62 & 0.17 & $<0.002$ & $1.87[1.32-2.63]$ \\
Thalassemia/sickle cell disease & 0.78 & 0.26 & 0.006 & $2.18[1.28-3.70]$ \\
Previous condition: drug addiction & 0.40 & 0.12 & 0.003 & $1.49[1.15-1.92]$ \\
Prenatal care in another service & 0.25 & 0.09 & 0.010 & $1.28[1.07-1.53]$ \\
Age (35-49 years $\times$ 20-34) & 0.22 & 0.08 & 0.007 & $1.25[1.07-1.45]$ \\
Previous condition: obesity & -0.52 & 0.14 & $<0.002$ & $0.59[0.44-0.80]$ \\
Marital status (no steady partner) & -0.65 & 0.14 & $<0.001$ & $0.52[0.39-0.69]$ \\
Constant & -3.51 & 0.22 & $<0.001$ & \\
\hline
\end{tabular}

$\mathrm{PR}_{\mathrm{adj}}$ : prevalence ratio adjusted by cluster effect and also by variables shown to be significant in the final model.

Multiple Poisson regression, controlled by: Age 2 (35-49 years: 1/ 20-34: 0); Ethnicity (Nonwhite: 1/Other: 0); Schooling (up to primary school: 1/Other: 0); Marital status (no steady partner: 1/Other: 0); BMI (Low weight, adequate: 0/overweight, obesity: 1); Prenatal care at the same service (Absent: $1 /$ Present: 0 ); Previous clinical conditions (Yes: 1/No: 0); Chronic hypertension (Yes: 1/No: 0); Obesity (Yes: 1/No: 0); Low weight (Yes: 1/No: 0); Diabetes mellitus (Yes: 1/No: 0); Smoking (Yes: 1/No: 0); Cardiac diseases (Yes: 1/No: 0); Respiratory diseases (Yes: 1/No: 0); Renal diseases (Yes: 1/No: 0); Thalassemia/sickle cell anemia (Yes: 1/No: 0); HIV/ AIDS (Yes: 1/No: 0); Thyroid diseases (Yes: 1/No: 0); Neurologic diseases/epilepsy (Yes: 1/No: 0); Collagenosis (Yes: 1/No: 0); Neoplasms (Yes: 1/No: 0); Other preexisting condition (Yes: 1/No: 0); Drug addiction (Yes: 1/No: 0); Parity (0/ $\geq 1$ : 1); Previous abortion (Yes: 1/No: 0); Previous Cesarean section (Yes: 1/No: 0); Time since last delivery (years); Number of prenatal visits (up to 5: 0/ $\geq 6: 1$ ); hemorrhagic conditions (Yes: 1/No: 0); Hypertensive conditions (Yes: $1 /$ No: 0 ); Infectious conditions (Yes: 1/No: 0); Clinical surgical conditions (Yes: 1/No: 0); Any delay (Yes: 1/No: 0).

Brazil, 2009-2010. 
For older women, aged 35 years or more, the risk of unfavorable outcome was significantly higher than in the reference population. In the extreme age group (age $>40$ years), this effect was even stronger. These findings have previously been reported by others $[2,14,16,17]$. Thus, as already known for maternal death [21], in the present study not only MMR, but also ratios of MNM increased swelled with increasing maternal age, except for the lower extreme of maternal age, again is in agreement with other published results [22-24]. The increased rate of MNM was probably the main finding of this analysis. According to the WHO [9], this indicator gives an estimate of the need to increment care and resources that are required to improve the results of obstetric care in certain regions. The differential gradient observed in different age groups could then also be used to prioritize investments and actions in groups identified as having a higher risk of MNM and MMR.

The comparison between several characteristics among the three maternal age groups of all women identified as having severe maternal morbidity in this study showed well-known differences among these groups, reflecting some unfavorable conditions for maternity in both extreme age groups, in comparison to an intermediate reproductive age. Thus, among adolescents with severe maternal morbidity, the non-white color was most frequent (poverty and greater vulnerability), lack of a steady partner and lower level of school education $[1,12,13]$. Adolescents may be at a disadvantage for not achieving the total number of school years and this inequality increases among non-white racial groups [25]. Similarly, nulliparity, vaginal delivery, low maternal weight and hypertension were more common as a cause of morbidity among adolescents. However, contrary to other studies [12], perinatal results were better in our cohort.

Obesity was significantly higher with increasing age. This finding confirms that the epidemic of obesity is the recent reality for the Brazilian population of reproductive aged women. Despite the lack of weight information for a large portion of the women in the study, more than $36 \%$ were above adequate weight even among adolescents; while $65 \%$ of women aged 35 years or older were overweight or obese. Obesity is not only a problem for the occurrence of SMM. It is also an issue that may possibly influence the future quality of life in these women. Nevertheless, obesity has also been previously identified as a risk factor for the occurrence of severe maternal morbidity [22,23]. The majority of preexisting morbid conditions were more prevalent among older women, especially hypertension, diabetes, heart and thyroid conditions. These conditions are more common with increasing age, and they have already been identified as risk factors for the occurrence of severe maternal morbidity [22].
Although a series of improvements have occurred in Brazil in economic, social and health terms in the last decades [26], maternal health is still characterized by relatively elevated mortality and morbidity rates. In addition to socioeconomic factors, our data highlights that extremely young and advanced maternal ages are associated with a higher risk of maternal complications that progress unfavorably to near-miss or maternal death. Special attention should be devoted to the provision of health services both for prenatal care and management during delivery and postpartum period.

\begin{abstract}
Abbreviations
Cl: Confidence intervals; ELB: Estimated number of live births; ICC: Intraclass correlation coefficients; LB: Live births; MD: Maternal death; MMR: Maternal mortality ratio; MNM: Maternal near miss; MNM:MD: The ratio between MNM and MD; MNMR: Maternal near-miss ratio; NM: Near miss; PLTC: Potentially life-threatening conditions; PR: Prevalence ratio; PSUm: Primary Sampling Units; SE: Standard error; SMM: Severe maternal morbidity; SMOR: Severe maternal outcome ratio; WHO: World Health Organization.
\end{abstract}

\section{Competing interests}

All authors declare that they have no competing interests.

\section{Authors' contributions}

The idea for the study and this specific analytical approach arose in a group discussion among all the authors. Analyses were planned and performed by FCO, FGS, JLPS, JGC and MHS. The first version of the manuscript was drafted by $\mathrm{FCO}$, and then complemented with suggestions from all the others, and mainly FGC, JLPS, JGC, and RCP. All authors contributed to the development of the study protocol, read and approved the final version of the manuscript.

\section{Funding}

This study was funded by CNPq/DECIT (The National Research Council and the Department of Science and Technology of the Brazilian Ministry of Health), grant number 402702/2008-5. The content is solely the responsibility of the authors and does not necessarily represent the official views of CNPq. It did not influence the content of the manuscript. This publication was supported by Fundacão de Amparo à Pesquisa do Estado de São Paulo (FAPESP), process number: 2014/03200-3.

\section{Brazilian Network for the Surveillance of Severe Maternal Morbidity} Group

Rodrigo S. Camargo, Vilma Zotareli, Lúcio T. Gurgel, Eliana M Amaral, Lale Say, Robert C Pattinson, Marilza V Rudge, Iracema M Calderon, Maria V Bahamondes, Danielly S Santana, Simone P Gonçalves, Olímpio B Moraes Filho, Simone A Carvalho, Francisco E Feitosa, George N Chaves, lone R Brum, Gloria C Saint'Ynes, Carlos A Menezes, Patricia N Santos, Everardo M Guanabara, Elson J Almeida Jr, Joaquim L Moreira, Maria R Sousa, Frederico A Peret, Liv B Paula, Luiza E Schmaltz, Cleire Pessoni, Leila Katz, Adriana Bione, Antonio C Barbosa Lima, Edilberto A Rocha Filho, Melania M Amorim, Debora Leite, Ivelyne Radaci, Marilia G Martins, Frederico Barroso, Denis J Nascimento, Cláudio S Paiva, Moises D Lima, Djacyr M Freire, Roger D Rohloff, Simone M Rodrigues, Sergio M Costa, Lucia C Pfitscher, Adriana G Luz, Daniela Guimaraes, Gustavo Lobato, Marcos Nakamura-Pereira, Eduardo Cordioli, Alessandra Peterossi, Cynthia D Perez, Jose C Peraçoli, Roberto A Costa, Nelson L Maia Filho, Jacinta P Matias, Silvana M Quintana, Elaine C Moises, Fátima A Lotufo, Luiz E Carvalho, Carla B Andreucci, Elvira Zanetti, Márcia M Aquino, Maria H Ohnuma, Rosiane Mattar and Felipe F

Campanharo.

\section{Author details}

${ }^{1}$ Department of Obstetrics and Gynecology, University of Campinas, Rua Alexander Fleming, 101, 13083-881 Campinas, SP, Brazil. Department of Gynecology and Obstetrics, Federal University of Paraná, Curitiba, PR, Brazil. ${ }^{3}$ Cemicamp: Centre for Research on Reproductive Health of Campinas, Campinas, Brazil. 
Received: 31 July 2013 Accepted: 10 February 2014

Published: 20 February 2014

\section{References}

1. Ham P, Allen C: Adolescent health screening and counseling. Am Fam Physician 2012, 86(12):1109-1116.

2. Jacobsson B, Ladfors L, Milsom I: Advanced maternal age and adverse perinatal outcome. Obstet Gynecol 2004, 104:727-733.

3. Lamminpää R, Vehviläinen-Julkunen K, Gissler M, Heinonen S: Preeclampsia complicated by advanced maternal age: a registry-based study on primiparous women in Finland 1997-2008. BMC Pregnancy Childbirth 2012, 12:47.

4. Pattinson RC, Hall M: Near misses: an useful adjunct to maternal death enquiries. Br Med Bull 2003, 67:231-243.

5. Cecatti JG, Souza JP, Parpinelli MA, Haddad SM, Camargo RS, Pacagnella RC, Silveira C, Zanardi DT, Costa ML, Silva JL P e, Passini R Jr, Surita FG, Sousa MH, Calderon IM, Say L, Pattinson RC, Brazilian Network for Surveillance of Severe Maternal Morbidity: Brazilian network for the surveillance of maternal potentially life threatening morbidity and maternal near-miss and a multidimensional evaluation of their long term consequences. Reprod Health 2009, 6:15.

6. Pattinson R, Say L, Souza JP, Van Den Broek N, Rooney C, WHO Working Group on Maternal Mortality and Morbidity Classifications: WHO maternal death and near-miss classifications. Bull World Health Org 2009, 87(10):734.

7. Cecatti JG, Souza JP, Parpinelli MA, Sousa MH, Amaral E: Research on severe maternal morbidities and near-misses in Brazil: what we have learned. Reprod Health Matters 2007, 15(30):125-133.

8. Say L, Souza JP, Pattinson RC, WHO working group on Maternal Mortality and Morbidity classifications: Maternal near miss-towards a standard tool for monitoring quality of maternal health care. Best Practice Res Clin Obstet Gynaecol 2009, 23(3):287-296.

9. WHO, World Health Organization: Evaluating the Quality of Care for Severe Pregnancy Complications: The WHO Near-Miss Approach for Maternal Health Geneva: WHO; 2011. http://whqlibdoc.who.int/publications/2011/ 978924150222_eng.pdf.

10. Cecatti JG, Souza JP, Oliveira Neto AF, Parpinelli MA, Sousa MH, Say L, Pattinson RC: Pre-validation of the WHO organ dysfunction based criteria for identification of maternal near miss. Reprod Health 2011, 8:22.

11. Tunçalp O, Hindin MJ, Souza JP, Chou D, Say L: The prevalence of maternal near miss: a systematic review. BJOG 2012, 119(6):653-661.

12. Conde-Agudelo A, Belizán JM, Lammers C: Maternal-perinatal morbidity and mortality associated with adolescent pregnancy in Latin America: cross-sectional study. Am J Obstet Gynecol 2005, 192(2):342-349.

13. WHO - World Health Organization: Why is Giving Special Attention to Adolescents Important for Achieving Millennium Development Goal 5? Fact Sheet, WHO/MPS/ 08.14. Adolescent Pregnancy. Geneva: WHO; 2008. http://www.gfmer.ch/SRHCourse-2010/adolescent-sexual-reproductive-health/WHO-adolescents-MDG52008.html.

14. Lampinen $R$, Vehviläinen-Julkunen $K$, Kankkunen P: A review of pregnancy in women over 35 years of age. Open Nurs J 2009, 3:33-38.

15. Koyanagi A, Zhang J, Dagvadorj A, Hirayama F, Shibuya K, Souza JP, Gülmezoglu AM: Macrosomia in 23 developing countries: an analysis of a multicountry, facility-based, cross-sectional survey. Lancet 2013, 381(9865):476-483.

16. Bayrampour $\mathrm{H}$, Heaman M: Advanced maternal age and the risk of cesarean birth: a systematic review. Birth 2010, 37:219-226.

17. Cleary-Goldman J, Malone FD, Vidaver J, Ball RH, Nyberg DA, Comstock CH, Saade GR, Eddleman KA, Klugman S, Dugoff L, Timor-Tritsch IE, Craigo SD, Carr SR, Wolfe HM, Bianchi DW, D'Alton M, FASTER Consortium: Impact of maternal age on obstetric outcome. Obstet Gynecol 2005, 105(5 Pt 1):983-990.

18. Haddad SM, Cecatti JG, Parpinelli MA, Souza JP, Costa ML, Sousa MH, Surita FG, Pinto E, Silva JL, Pacagnella RC, Camargo RS, Bahamondes MV, Zotareli V, Gurgel LT, Say L, Pattinson RC, National Network for the Surveillance of Severe Maternal Morbidity Group: From planning to practice: building the national network for the Surveillance of Severe Maternal Morbidity. BMC Public Health 2011, 11:283.

19. Haddad SM, Sousa MH, Cecatti JG, Parpinelli MA, Costa ML, Souza JP, Brazilian Network for Surveillance of Severe Maternal Morbidity Group: Intraclass correlation coefficients in the Brazilian network for surveillance of severe maternal morbidity study. BMC Pregnancy Childbirth 2012, 12(1):101.
20. Brazil, Ministry of Health. DATASUS: System of Information on Live Births (SINASC): Number of Live Births by age and Geographical Regions, Brazil 2009. http://tabnet.datasus.gov.br/cgi/tabcgi.exe?sinasc/cnv/nvuf.def.

21. Lisonkova S, Liu S, Bartholomew S, Liston RM, Joseph KS, Maternal Health Study Group of the Canadian Perinatal Surveillance System: Temporal trends in maternal mortality in Canada II: estimates based on hospitalization data. J Obstet Gynaecol Can 2011, 33(10):1020-1030.

22. Gray KE, Wallace ER, Nelson KR, Reed SD, Schiff MA: Population-based study of risk factors for severe maternal morbidity. Paediatr Perinat Epidemiol 2012, 26(6):506-514.

23. Zwart JJ, Richters JM, Ory F, de Vries JI, Bloemenkamp KW, van Roosmalen J: Severe maternal morbidity during pregnancy, delivery and puerperium in the Netherlands: a nationwide population-based study of 371,000 pregnancies. BJOG 2008, 115(7):842-850.

24. Souza JP, Cecatti JG, Parpinelli MA, Sousa MH, Lago TG, Pacagnella RC, Camargo RS: Maternal morbidity and near miss in the community: findings from the 2006 Brazilian demographic health survey. BJOG 2010, 117(13):1586-1592.

25. Victora CG, Matijasevich A, Silveira MF, Santos IS, Barros AJ, Barros FC: Socio-economic and ethnic group inequities in antenatal care quality in the public and private sector in Brazil. Health Policy Plann 2010, 25:253-261.

26. Victora CG, Aquino EM, do Carmo Leal M, Monteiro CA, Barros FC, Szwarcwald CL: Maternal and child health in Brazil: progress and challenges. Lancet 2011, 377 (9780):1863-1876.

\section{doi:10.1186/1471-2393-14-77}

Cite this article as: Oliveira Jr et al.: Severe maternal morbidity and maternal near miss in the extremes of reproductive age: results from a national cross- sectional multicenter study. BMC Pregnancy and Childbirth $201414: 77$.

\section{Submit your next manuscript to BioMed Central and take full advantage of:}

- Convenient online submission

- Thorough peer review

- No space constraints or color figure charges

- Immediate publication on acceptance

- Inclusion in PubMed, CAS, Scopus and Google Scholar

- Research which is freely available for redistribution

Submit your manuscript at www.biomedcentral.com/submit
C Biomed Central 